INPLASY

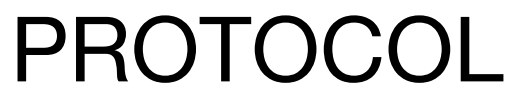

To cite: Rodrigues-Santana et al. The Effects of Whole Body Muscle Stimulation on body composition and strength parameters. A protocol for systematic review and meta analysis. Inplasy protocol 202120050. doi: 10.37766/inplasy2021.2.0050

Received: 16 February 2021

Published: 16 February 2021

Corresponding author:

Luiz Rodrigues-Santana

luiz88santana@hotmail.com

Author Affiliation:

University of Extremadura

Support: Award Number:

18.KG.00.

Review Stage at time of this submission: The review has not yet started.

Conflicts of interest: None declared.

\section{The Effects of Whole Body Muscle Stimulation on body composition and strength parameters. A protocol for systematic review and meta analysis}

Rodrigues-Santana, L1; Adsuar, JC2; Louro, H33; Pérez-Gómez, J4; Hernández-Mocholí, MA5; Carlos-Vivas, J6; Gomez-Campos, R7; Correia de Campos, LC8.

Review question / Objective: What is the effect of the whole body electrical stimulation compared to other training methods or control group in the body composition and strength parameters?

Condition being studied: Body Composition, Muscle Mass, Fat Mass, Muscle Strength, Force.

Information sources: Studies will be searched in the following electronic databases: PubMed, Web of Science, Scopus, SPORTDiscus and EMBASE until November 30 only in English language, without limitations of publication status. All randomized clinical trials will be considered

INPLASY registration number: This protocol was registered with the International Platform of Registered Systematic Review and Meta-Analysis Protocols (INPLASY) on 16 February 2021 and was last updated on 16 February 2021 (registration number INPLASY202120050).

\section{INTRODUCTION}

Review question / Objective: What is the effect of the whole body electrical stimulation compared to other training methods or control group in the body composition and strength parameters?
Condition being studied: Body Composition, Muscle Mass, Fat Mass, Muscle Strength, Force.

\section{METHODS}

Participant or population: Trained and untrained adults (over 18 years old). 
Intervention: The subjects were exposed to a Whole Body Electro Muscle Stimulation training program. WB-EMS is a timeefficient method of training that activates simultaneously several muscle groups. It uses differents current parameters (frequency, wave length, contraction time, rest time and intensity) superimposed on conventional training exercises.

Comparator: - No exercise/control. - Any type of exercise including strengthening exercise: weight training and other resistive exercises. - "placebo group" with the same training program as the WB-EMS group. Same volume and intensity applied.

Study designs to be included: We will include randomised control trails (RCT) to assess the effects of the WB-EMS programs.

Eligibility criteria: This study will include randomized clinical trials investigating the effects of whole-body electromyostimulation training on body composition and strength indicators.

Information sources: Studies will be searched in the following electronic databases: PubMed, Web of Science, Scopus, SPORTDiscus and EMBASE until November 30 only in English language, without limitations of publication status. All randomized clinical trials will be considered.

Main outcome(s): Outcome related to body composition Fat-free mass, fat mass, body mass index, muscle mass, lean mass measured by electrical bioimpedance, dual-energy x-ray absorptiometry (DEXA), skin folds or anthropometric measurements.

Additional outcome(s): Muscle strength by voluntary isometric muscular strength measurement.

Quality assessment / Risk of bias analysis: Risk bias will be investigated by two independent researchers (LSR and MAHM) using the PEDro scale. GRADE System will be use for Classification of Quality of
Evidence and Strength of Recommendation.

Strategy of data synthesis: We will use I $^{2}$ statistic to assessed the heterogeneity. We will do the Funnel Plot to asses publication bias. If it is possible we will do metaregression analysis. We will include both random-effects model and fixed-effects.

Subgroup analysis: Subgroup analysis will be done by: - Male/Famale - Trained and Untrained.

Sensitivity analysis: We will do a sensitivity analysis to examine the robustness and stability of the synthesized results by eliminating low quality studies.

Language: English.

Country(ies) involved: Spain.

Keywords: WBody Composition; B-EMS; Electromyostimulation; Strength; Fat Mass; Lean Body Mass.

Contributions of each author:

Author 1 - Luiz Rodrigues-Santana Initiator of the Project. Development of the review question and condition being studied. Responsible for protocol registration.

Email: luiz88santana@hotmail.com

Author 2 - Jose C Adsuar - Writing the protocol: Eligibility criteria and Search Strategy.

Email: carmelo.adsuar@gmail.com

Author 3 - Hugo Louro - Writing the protocol. Development of the selection criteria and risk of bias.

Email: hlouro@esdrm.ipsantarem.pt

Author 4 - Jorge Pérez-Gómez - Writing the Protocol. The author developed Study Selection and Data Collection Section.

Email: jorgepg100@gmail.com

Author 5 - Miguel A Hernández-Mocholí The author developed the data synthesis, Subgroup Analysis and Sensitivity Analysis Section.

Email: mhmocholi@unex.es

Author 6 - Jorge Carlos-Vivas - The author developed the search strategy.

Email: jorge.carlosvivas@gmail.com 
Author 7 - Rossana Gomez-Campos - The author developed the Discussion and Limitation Section.

Email:rossaunicamp@gmail.com

Author 8 - Luis Castelli Correia de Campos

- Responsible for reviewing the protocol.

Email: fcastelli@hotmail.com 